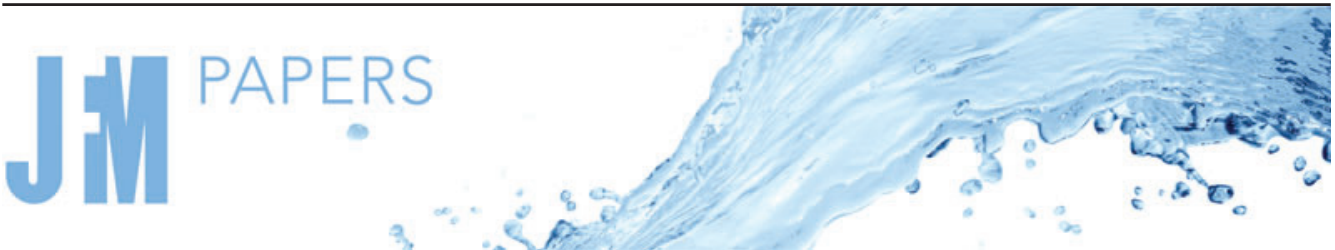

\section{Drag, diffusion and segregation in inertial granular flows}

\author{
Robbie S.J. Bancroft ${ }^{1}{ }_{\dagger}$ and Chris G. Johnson ${ }^{1}$ \\ ${ }^{1}$ Department of Mathematics and Manchester Centre for Nonlinear Dynamics, University of Manchester, \\ Oxford Road, Manchester M13 9PL, UK
}

(Received 11 November 2020; revised 14 May 2021; accepted 16 June 2021)

\begin{abstract}
Inter-species drag forces in granular flows play a central role in setting the speed and extent of segregation, a process that separates grains of different size or density. Here, we study this drag force in detail, using a novel configuration of discrete element simulations that allows us to completely characterise the drag in inertial granular flows by studying it in a uniform environment. By applying opposing forces to grains in monodisperse and size-bidisperse shear flows, we show that the strength of the drag force scales as $I^{-7 / 4}$, where $I$ is the granular inertial number, and propose a model that explains this scaling by relating the strength of drag to grain velocity fluctuations. These findings suggest that much of the previously observed dependence of the segregation rate on the local shear rate and pressure in dense free-surface flows is due to variation in the strength of the inter-species drag, rather than the strength of forces that drive segregation.
\end{abstract}

Key words: granular mixing, dry granular material, avalanches

\section{Introduction}

Granular segregation occurs routinely whenever a mixture of non-identical grains is sheared. This segregation generates a small relative motion between grains of different size, density or shape, which over time causes demixing and a spatially inhomogeneous composition of the granular material. The process of segregation is of fundamental importance in both industrial granular flows, where it hinders uniform mixing of bulk solids (see e.g. Fan et al. 2014), and in natural granular avalanches, where the segregation of large boulders to the front and sides of the flow prevents lateral spreading but promotes flow instabilities and a longer runout (Johnson et al. 2012).

Segregation occurs through various mechanisms, and across a wide range of parameter regimes, in shear flows (Savage \& Lun 1988), vibrated systems (Ahmad \& Smalley 1973)

$\dagger$ Email address for correspondence: robbie.bancroft@manchester.ac.uk 
and quasi-static granular flows (Chassagne et al. 2020), and in both mixtures of many types of grains and systems that are monodisperse apart from a single large 'intruder' (e.g. van der Vaart et al. 2018; Jing et al. 2020). This paper focuses on granular flows in the inertial regime - that is, dense, liquid-like flows in which grain fluctuations are generated by shear, and where the rheology is independent of the inter-grain coefficient of restitution (Forterre \& Pouliquen 2008). While not applicable to dilute, vibrated or quasi-statically deforming granular systems, this inertial regime encompasses a wide range of dense granular flows (GDR-MiDi 2004). In particular, it includes dense free-surface granular flows, which are particularly efficient at segregating and separating grains of different species (Savage \& Lun 1988), and cause pronounced segregation in hoppers (Baxter et al. 1998), rotating drums (Gray \& Hutter 1997; Hill \& Tan 2014) and natural granular avalanches.

Even within the inertial regime, the micro-mechanical origins of segregation are still subject to debate; there is not yet a general theory that can predict the rate of segregation (that is, the speed at which different grain species percolate through one another) given the flow conditions and the properties of the grains. Nonetheless, continuum descriptions have been successful in describing the qualitative behaviour of segregating flows, and are able to make quantitative predictions when parameters are suitably chosen for a given flow configuration. Many such models are expressed in the framework of mixture theory (e.g. Gray \& Thornton 2005; Marks, Rognon \& Einav 2012; Fan et al. 2014; Hill \& Tan 2014; Tunuguntla, Bokhove \& Thornton 2014). In these theories, each species of grain $i$ is modelled as a separate fluid constituent with its own species fraction $\phi_{i}$, grain diameter $d_{i}$, intrinsic density $\rho_{i}^{*}$, extrinsic density $\rho_{i}=\phi_{i} \rho_{i}^{*}$ and velocity $\boldsymbol{u}_{i}$. The set of fractions $\left\{\phi_{i}\right\}$ represent the fractions of the solid material belonging to each species, with $0 \leqslant \phi_{i} \leqslant 1$ and $\sum_{i} \phi_{i}=1$. Bulk flow quantities, such as the bulk velocity $\boldsymbol{u}=\sum_{i} \phi_{i} \boldsymbol{u}_{i}$, are defined as a volume-weighted average of the constituent properties (e.g. Gray \& Thornton 2005).

Within these mixture theory models, each species obeys a mass balance equation

$$
\frac{\partial \rho_{i}}{\partial t}+\nabla \cdot\left(\rho_{i} \boldsymbol{u}_{i}\right)=0 .
$$

The flux $\rho_{i} \boldsymbol{u}_{i}$ is assumed be the result of an equilibrium of three forces acting on the $i$ th species (Gray \& Chugunov 2006). This force balance takes the form

$$
\boldsymbol{S}_{i}-\rho_{i} c\left(\boldsymbol{u}_{i}-\boldsymbol{u}\right)-\left(\sum_{j} \rho_{j}\right) c D \nabla \phi_{i}=0,
$$

where the first term, $S_{i}$, is a driving segregation force per unit volume and the second term is an inter-species drag force (assumed linear in the segregation velocity $\boldsymbol{u}_{s i}:=\boldsymbol{u}_{i}-\boldsymbol{u}$ ) with coefficient $c$, which opposes the relative motion between different granular species. The final term is an effective force arising from a diffusion with diffusivity $D$ (which is assumed to be normal, or Fickian).

In inertial granular flows, the segregation force $S_{i}$ and the segregation velocity $\boldsymbol{u}_{s i}$ that results from it are generated by spatial gradients in the flow. Analysis from kinetic theory (Larcher \& Jenkins 2015) and discrete element simulations (Staron \& Phillips 2015) suggest that gravity-induced hydrostatic gradients of pressure $p$ (as in Gray \& Thornton 2005) and gradients of granular temperature $T$ or kinetic stress (Hill \& Tan 2014) can all contribute to the driving segregation force $S_{i}$, as can gradients of shear stress (Guillard, Forterre \& Pouliquen 2016), although the relative importance of these contributions is difficult to assess (Staron \& Phillips 2015).

The segregation velocity can also depend on the local value of flow properties, through the drag force. In regions of uniform $\phi_{i}$, where the diffusive flux is zero, the force balance 


\section{Drag forces in granular segregation}

(1.2) implies that the segregation velocity is simply a balance of the segregation and drag forces,

$$
\boldsymbol{u}_{s i}=\boldsymbol{u}_{i}-\boldsymbol{u}=\frac{\boldsymbol{S}_{i}}{\rho_{i} c}=\frac{\boldsymbol{S}_{i}\left(\nabla p, \nabla T, \ldots, \dot{\gamma}, p,\left\{\phi_{i}\right\}, \ldots\right)}{\rho_{i} c\left(\dot{\gamma}, p,\left\{\phi_{i}\right\}, \ldots\right)} .
$$

Across multiple studies, the segregation velocity is observed to be approximately linear in the local shear rate $\dot{\gamma}$ (Marks et al. 2012; Fan et al. 2014; Larcher \& Jenkins 2015) and a decreasing function of the pressure $p$ (Golick \& Daniels 2009; Fry et al. 2018; Duan et al. 2020), although the precise form of these dependencies, and the mechanisms leading to them, are still uncertain.

In particular, it has not been shown whether dependencies of the segregation velocity on the local flow properties (such as $\dot{\gamma}, p$ ) come about through variation with these properties of the driving force $S_{i}$, the drag coefficient $c$ or both of these mechanisms.

In this paper we isolate the process of inter-species drag, and study it separately from the driving segregation force $S_{i}$. In doing so, we completely characterise the drag coefficient $c$ and its dependence on local flow properties for dense inertial flows of monodisperse and size-bidisperse grains, and provide a model that explains the scaling of these dependencies. In the absence of spatial gradients (a uniform flow) the driving segregation force $S_{i}$ is zero. Consequently, measurements of this force in experiments or discrete element simulations require the system to be spatially inhomogeneous. The inter-species drag, on the other hand, is not driven by gradients, and so can be studied in a spatially uniform flow, as we do here.

We measure the drag coefficient $c$ and characterise its dependence on the flow using discrete element method (DEM) simulations of grains in a gravity-free, steady, spatially uniform shear flow. The grains are partitioned into two distinct groups, one 'rising' species and one 'falling' species. In absence of gravity or spatial gradients, no segregating forces arise between these two species. Instead, we apply a small positive or negative 'buoyancy' force, respectively, to grains of each species. This force causes the grain species to percolate through each other at a rate controlled purely by the applied buoyancy force and the drag. By tracking the both the average and fluctuating motion of individual grains, we measure both this percolation velocity, and the diffusivity $D$, as a function of the local flow parameters. This drag-controlled percolation process is very similar to that occurring during the segregation of two different granular species, but is driven here by an imposed buoyancy force that allows a spatially uniform flow, rather than by the segregation force $S_{i}$ that results from spatial gradients.

The spatially inhomogeneous flows typically required to study granular segregation mean that dimensional analysis can only be applied globally to the entire system, and cannot be used to determine the local relationship between segregation velocity and flow properties that is usually desired. In contrast, because the system studied here is uniform, dimensional analysis can be used to considerably simplify the study of how the drag and diffusion depend on the local flow properties such as shear rate and pressure. With a combination of dimensional analysis and discrete element simulations, we are able to validate the form of the drag and diffusion terms in (1.2) and fully characterise $c$ and $D$.

In $\S 2$ we describe the DEM simulations and the complete set of parameters used. In $\S 3$ we reduce these parameters to the underlying dimensionless groups, and use our simulations to evaluate the dependence of the drag and diffusion on each of these. We initially consider monodisperse grains, and show that the drag coefficient $c$ scales with the granular inertial number $I$ to the power $-7 / 4$. We explain the origin of this scaling with a model that relates segregation velocity to the grain velocity fluctuations. We then demonstrate that this scaling also holds when a size ratio is introduced between the 


Parameter
(Large) grain diameter
Applied shear rate
Intrinsic grain density
Periodic box size
Contact normal stiffness
Contact tangential stiffness
Buoyancy acceleration
Contact friction coefficient
Contact restitution coefficient
Solids volume fraction
Rising grain fraction
Grain size ratio

Symbol
$d$
$\dot{\gamma}$
$\rho^{*}$
$H$
$k_{n}$
$k_{t}$
$b$
$\mu$
$\varepsilon$
$\Phi$
$\phi_{r}$
$s$

Values
$10^{-3}$
$0.1-10$
2500
$12 d$
$10^{3}$
$2 k_{n} / 7$
$10^{-4}-1$
$0.3,0.5,1.0$
$0.5,0.8$
$0.42-0.6$
$0.1-0.9$
$1-2$

Units

$$
\begin{gathered}
\mathrm{m} \\
\mathrm{s}^{-1} \\
\mathrm{~kg} \mathrm{~m}^{-3} \\
\mathrm{~m} \\
\mathrm{~N} \mathrm{~m}^{-1} \\
\mathrm{~N} \mathrm{~m}^{-1} \\
\mathrm{~m} \mathrm{~s}^{-2}
\end{gathered}
$$

Dimensionless

Dimensionless

Dimensionless

Dimensionless

Dimensionless

Table 1. Parameters used in the discrete element simulations. The first three parameters define the non-dimensionalisation and, in the inertial regime studied, the results are independent of the subsequent three. The final six parameters therefore govern the macroscopic system behaviour.

two species. We also show that the non-dimensional diffusivity is weakly dependent on I. In $\S 4$, we discuss how these findings inform models of granular segregation, and note that the variation of the drag with shear rate and pressure is able to account for much of the previously observed dependence of segregation rates on these quantities in free-surface flows. Finally we conclude in $\S 5$.

\section{DEM modelling}

We perform discrete element simulations to characterise the inter-species drag using the open-source software MercuryDPM (Weinhart 2020). The simulations involve twelve parameters (table 1), but, as we will show, for monodisperse inertial granular flows these reduce to just two dimensionless groups that describe the percolation of the two species. Grains are simulated as spheres of intrinsic density $\rho^{*}$ and diameter $d$. In bidisperse mixtures the grain diameters are $d$ and $d / s$, where $s$ is the grain size ratio. The grains satisfy Newton's laws of motion and interact via contact forces when they overlap one another. We use the common linear spring-dashpot contact force model (Cundall \& Strack 1979), with the magnitude of the normal and tangential forces acting on contacting grains being

$$
F_{n}=-k_{n} \delta_{n}-\eta_{n} \dot{\delta}_{n} \quad \text { and } \quad F_{t}=\min \left(-k_{t} \delta_{t}, \mu F_{n}\right),
$$

respectively, where $\delta_{n, t}$ are the normal and tangential overlaps between the grains, $k_{n, t}$ are the normal and tangential spring stiffness, $\eta_{n}$ is the normal dissipation and $\mu$ is the coefficient of sliding friction between two grains. The normal dissipation is set by specifying a coefficient of restitution $\varepsilon$ between two colliding grains. The grain stiffness chosen is sufficiently large that the flows are in the inertial regime, typical of dense granular avalanches, where results are independent of both stiffness parameters $k_{n}$ and $k_{t}$ (Silbert et al. 2001; Chialvo, Sun \& Sundaresan 2012).

The grains are simulated in a three-dimensional domain with periodic boundary conditions in $x$ and $y$, and Lees Edwards boundary conditions (Lees \& Edwards 1972) imposing a uniform simple shear with shear rate $\dot{\gamma}$ in the $x z$ plane (figure 1). No gravitational force is imposed. The periodic domain has side length $H=12 d$; we have verified that our results are insensitive to this choice of $H$. By varying the number of 


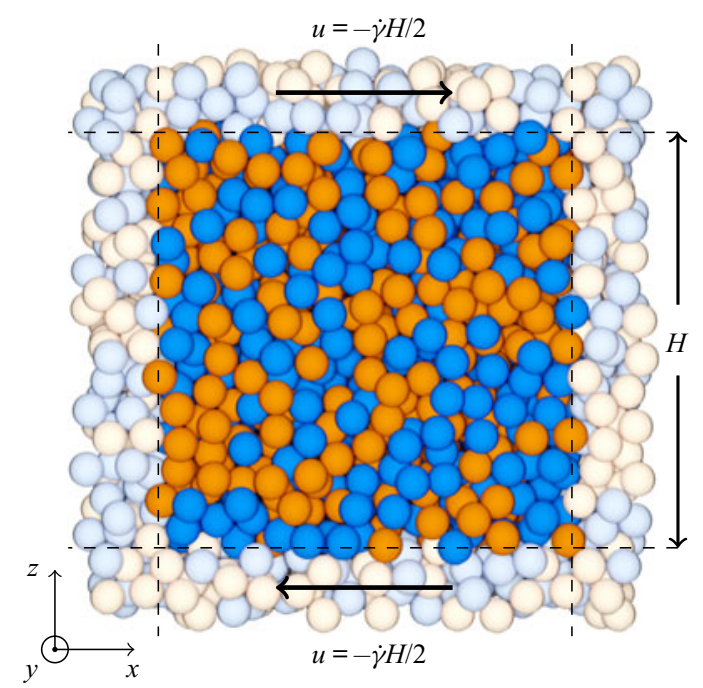

Figure 1. Cross-section of the three-dimensional Lees Edwards shear cell, with one periodic unit highlighted. The rising species of grains is coloured blue and falling species coloured orange.

large and small grains in the box $N_{l}$ and $N_{s}$, the solids volume fraction $\Phi=\pi d^{3}\left(N_{l}+\right.$ $\left.N_{S} / s^{3}\right) /\left(6 H^{3}\right)$ can be specified. The grains are initially placed at random positions and velocities within the domain, and the system is allowed to evolve to a uniform statistically steady state.

To induce percolation in the shear cell, the grains are assigned to rising and falling species, with the fraction of grains in the rising species denoted by $\phi_{r}$ and the fraction in the falling species by $\phi_{f}=1-\phi_{r}$. An acceleration $b_{i}$ is applied in the $z$ direction to each grain of species $i$, where $i=r, f$. Introducing a parameter $b$ that sets the relative acceleration between two species $\left(b_{r}-b_{f}=2 b\right)$ and requiring that the total force applied to the system is zero $\left(\rho_{r} b_{r}+\rho_{f} b_{f}=0\right)$, we obtain $b_{i}= \pm 2 b\left(1-\phi_{i}\right)$. The total force per unit volume applied to a species is then

$$
S_{i}=\rho_{i} b_{i} \hat{z}= \pm 2 b \rho^{*} \phi_{i}\left(1-\phi_{i}\right) \hat{z}, \quad i=r, f .
$$

The applied buoyancy force causes the two species to percolate through one another at a velocity $\boldsymbol{u}_{p i}=w_{p i} \hat{z}$. When the volume of rising and falling grains are equal $\left(\phi_{r}=\phi_{f}=\right.$ $0.5)$ the applied force reduces to $S_{i}= \pm b \rho_{i} \hat{z}$. We choose to measure the percolation velocity of the (larger) rising grains $w_{p r}$, but have verified that, as expected, $\phi_{r} w_{p r}+$ $\phi_{f} w_{p f}=0$.

\section{Dimensional analysis and simulation results}

We initially consider monodisperse grains, generalising to size-bidisperse mixtures in $\S 3.3$. In a uniform shear flow of identical spherical grains, there are just three dimensional parameters: the grain diameter $d$, the intrinsic grain density $\rho^{*}$ and the applied shear rate $\dot{\gamma}$, from which no dimensionless group can be formed. In addition, there are three dimensionless parameters: the solids volume fraction $\Phi$, and friction and restitution coefficients of the contact law, $\mu$ and $\varepsilon$, respectively. Dimensional analysis then requires that the pressure $p$ in such a granular shear flow is given by

$$
p=\rho^{*} d^{2} \dot{\gamma}^{2} \mathcal{F}(\Phi, \mu, \varepsilon),
$$




\section{R.S.J. Bancroft and C.G. Johnson}

for some dimensionless function $\mathcal{F}$. Our addition of a buoyancy force to this system introduces an additional dimensionless parameter,

$$
B=\frac{b_{r}}{d \dot{\gamma}^{2}},
$$

which can be interpreted as the square of the ratio of the time taken for a grain to move over its own diameter due to the buoyancy force, $\left(d / b_{r}\right)^{1 / 2}$, to the shear time scale $1 / \dot{\gamma}$. Assigning each grain to one of two species also introduces a new dimensionless parameter, $\phi_{r}$, the fraction of grains in the rising species. Accordingly, the pressure and percolation velocity in the shear cell are given by

$$
p=\rho^{*} d^{2} \dot{\gamma}^{2} \mathcal{F}\left(\Phi, B, \phi_{r}, \mu, \varepsilon\right) \quad \text { and } \quad w_{p r}=d \dot{\gamma} \mathcal{G}\left(\Phi, B, \phi_{r}, \mu, \varepsilon\right),
$$

for unknown functions $\mathcal{F}$ and $\mathcal{G}$. In the remainder of this section, we will use the DEM simulations to completely characterise the dependence of $\mathcal{F}$ and $\mathcal{G}$ on their parameters.

\subsection{Effect of the buoyancy force}

To examine the effect the buoyancy force has on the shear cell, we measure the pressure and percolation velocity in the shear cell at a range of dimensionless buoyancies $B$ and solids volume fractions $\Phi$. The percolation velocity is measured by tracking the mean distance moved by each grain through the steady state simulation time. The pressure is measured by coarse graining the contact and kinetic stresses between grains (using the method of Weinhart, Luding \& Thornton 2013) to find a continuum pressure field, which is then spatially and temporally averaged.

We find that the percolation velocity $w_{p r}$ is linear in the dimensionless buoyancy force $B$ (figure $2 a$ ), which is consistent with the assumption of a linear inter-species drag in (1.2). Measurements of the percolation velocity collapse across a range of volume fractions when the non-dimensional percolation speed $w_{p r} /(d \dot{\gamma})$ is plotted against

$$
\tilde{B}=B\left(\Phi_{c}-\Phi\right)^{7 / 4}
$$

where $\Phi_{c}$ is a critical volume fraction. The definition of $\Phi_{c}$ and the origin of the scaling power $7 / 4$ are discussed below. The collapse of the percolation velocity in (figure $2 a$ ) is clearest when $10^{-3} \leqslant \tilde{B} \leqslant 0.02$. Over the time scale of our simulations, the condition $\tilde{B} \geqslant$ $10^{-3}$ means that the percolation displaces grains by a much greater distance than random fluctuations (evidenced by the red bars on figure $2 a$ ). When $\tilde{B} \lesssim 0.02$ the measured pressure in the simulation, plotted in figure $2(b)$, is very similar to its value when there is no applied force, $\tilde{B}=0$, indicating that the buoyancy force is not significantly modifying the underlying shear flow. This is the appropriate regime for segregation in inertial granular flows, where segregation processes are typically much weaker than the underlying shear flow, and all further simulations presented therefore use buoyancy strengths which satisfy $10^{-3} \leqslant \tilde{B} \leqslant 10^{-2}$.

Since the pressure is unaffected by the imposed buoyancy force when $\tilde{B} \lesssim 0.02$, our observations of the pressure in this regime match the behaviour previously reported in non-segregating inertial granular flows (da Cruz et al. 2005; Chialvo et al. 2012). Namely, 

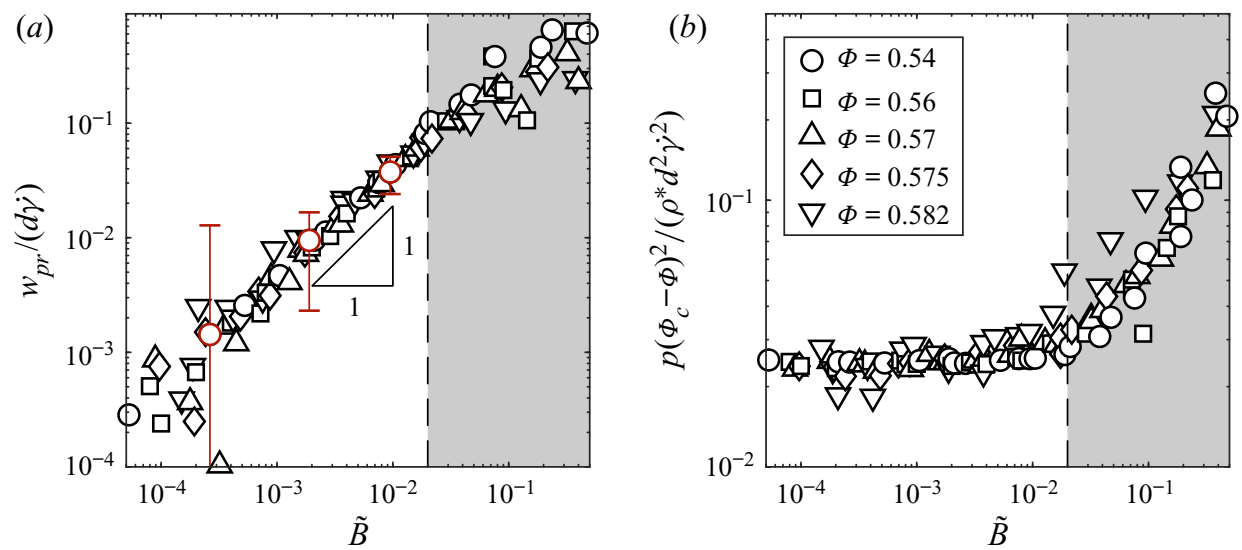

Figure 2. Collapse of ( $a$ ) dimensionless percolation velocity and $(b)$ the dimensionless scaled pressure, against scaled dimensionless buoyancy $\tilde{B}=B\left(\Phi_{c}-\Phi\right)^{7 / 4}$, with $\phi_{r}=0.5, \mu=0.5$ and $\varepsilon=0.8$. Red bars in $(a)$ show the standard deviation of $\sim 9000$ consecutive measurements of $w_{p r} /(d \dot{\gamma})$; white symbols show the mean of these measurements.

the measured pressure scales with the volume fraction $\Phi$ according to

$$
p \sim \frac{\rho^{*} \dot{\gamma}^{2} d^{2}}{\left(\Phi-\Phi_{c}(\mu)\right)^{2}},
$$

consistent with $(3.3 a)$, where $\Phi_{c}$ is a critical packing fraction that depends only on the grain friction coefficient $\mu$. Equivalently, the inertial number

$$
I=\frac{d \dot{\gamma}}{\sqrt{p / \rho^{*}}} \sim \Phi_{c}(\mu)-\Phi
$$

is a function of $\Phi$, as verified in the numerical simulations (figure $3 a$ ). This relationship allows the inertial number $I$, a dimensionless group involving a simulation output $p$, to be used as a proxy for a dimensionless input parameter of the system, $\Phi$, which varies only slightly in dense granular flows (GDR-MiDi 2004). Eliminating $\Phi$ in this manner makes explicit the relationship between percolation velocity and the flow properties $p$ and $\dot{\gamma}$, which, particularly in free-surface granular flows, are easier to infer experimentally than the volume fraction $\Phi$.

\subsection{Percolation velocity and drag coefficient}

Having confirmed that the percolation velocity $w_{p r}$ is linear in $B$ (figure $2 a$ ), and that the inertial number $I$ can substitute for the volume fraction $\Phi$ (figure $3 a$ ), our expression for the percolation velocity $(3.3 b)$ reduces to

$$
w_{p r}=\frac{b_{r}}{\dot{\gamma}} \mathcal{G}\left(I, \phi_{r}, \mu, \varepsilon\right) .
$$

We reaffirm the linearity in $B$ and examine the dependence of $w_{p r}$ on the remaining parameters in (3.7) by performing the shear cell simulations using five different combinations of friction and restitution coefficients, and over a range of shear rates, buoyancy strengths and solids volume fractions, which give a wide range of inertial numbers. As illustrated in figure 3(c), the percolation velocity collapses extremely well 

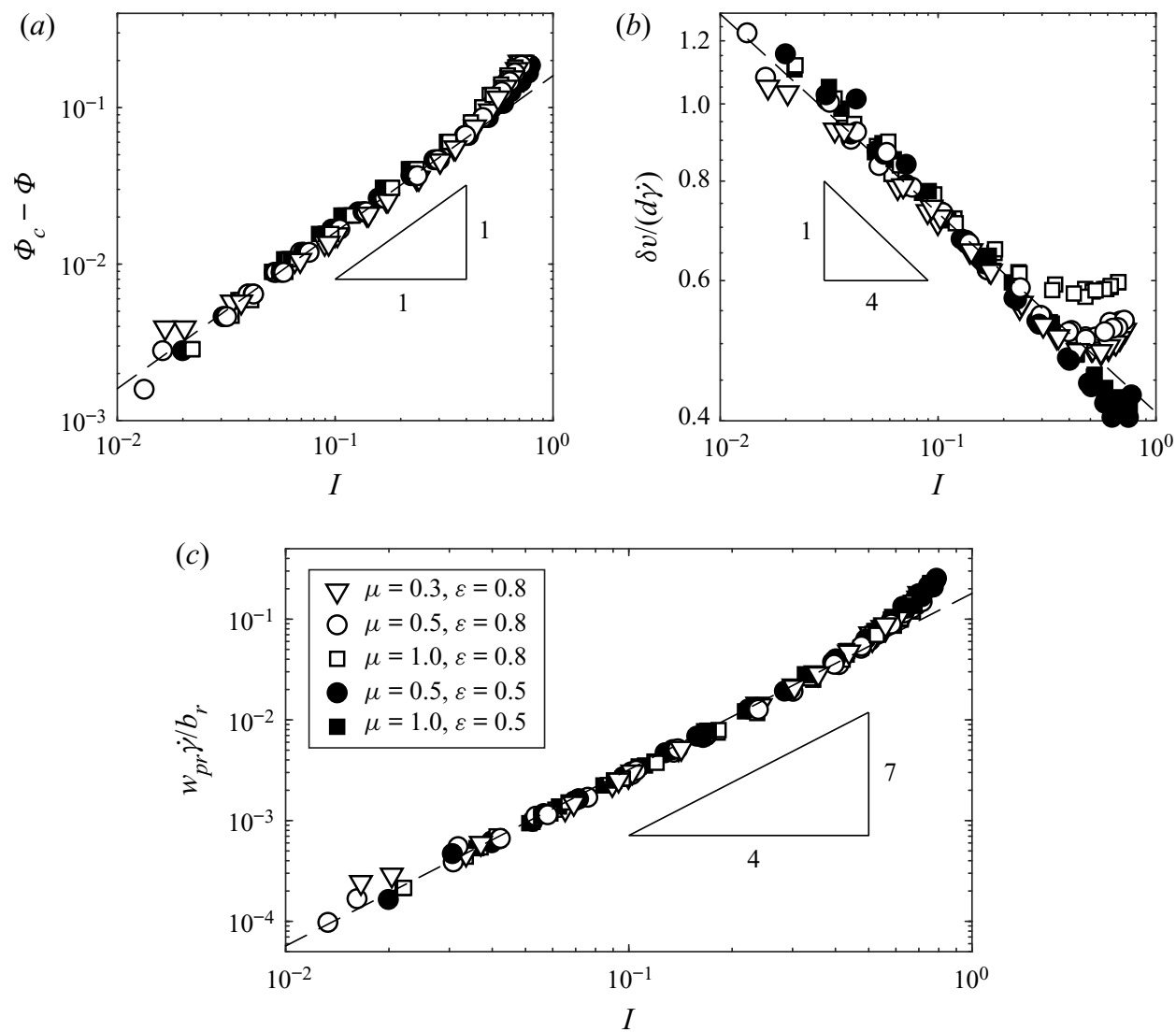

Figure 3. Collapse over inertial number, grain friction and restitution coefficient of (a) distance from critical packing fraction, $(b)$ magnitude of velocity fluctuations and $(c)$ non-dimensionalised percolation velocity. The fraction of rising grains is $\phi_{r}=1 / 2$, and the grains are monodisperse, $s=1$.

against the inertial number for all $B, \mu, \epsilon$, and exhibits a clear power-law scaling with $I$, for $I \lesssim 0.5$, reducing (3.7) to

$$
w_{p r}=\frac{b_{r}}{\dot{\gamma}} \mathcal{G}\left(\phi_{r}\right) I^{1.74 \pm 0.11} \approx \frac{b_{r}}{\dot{\gamma}} \mathcal{G}\left(\phi_{r}\right) I^{7 / 4} .
$$

The rising grain fraction $\phi_{r}$ has no discernible effect on the percolation velocity (figure 4), meaning that $\mathcal{G}\left(\phi_{r}\right)$ is a constant, with a value $\kappa=0.17 \pm 0.03$ measured directly from the simulations. Through (1.3), this provides an expression for the drag coefficient

$$
c=\frac{\left\|\boldsymbol{S}_{r}\right\|}{\rho_{r}\left\|\boldsymbol{u}_{p r}\right\|}=\frac{b_{r}}{w_{p r}}=\frac{\dot{\gamma}}{\kappa I^{7 / 4}} .
$$

To understand the origin of the observed power law $c \sim I^{-1.74}$ and the closeness of its exponent to $-7 / 4$, we consider a model of hard grains interacting through instantaneous pairwise collisions. This approach extends that of Bagnold (1954), who reasoned that granular pressure arose from the momentum transfer occurring at these instantaneous collisions. Although the assumption of pairwise collisions does not necessarily hold for dense flows with low inertial numbers, we find that the scalings for the drag coefficient 


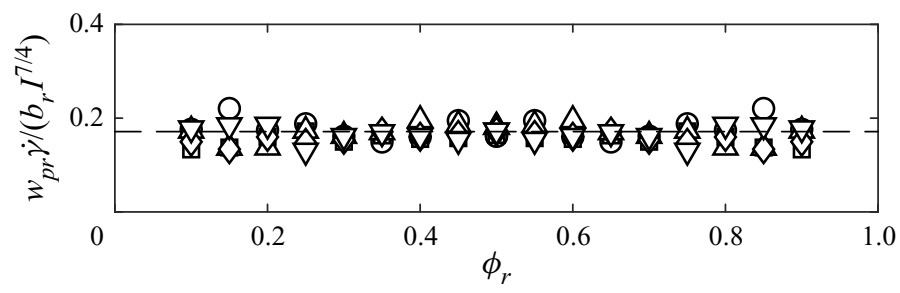

Figure 4. Dependence of percolation velocity on rising grain fraction. Symbol shapes indicate volume fractions $\Phi \in[0.54,0.582]$ as in figure $2 ; \mu=0.5, \varepsilon=0.8, s=1$.

obtained from this approach (like the scalings for the pressure deduced by Bagnold 1954) remain valid well into this dense regime.

Each grain collides with another at rate characterised by the mean free time $t_{m f}$. Denoting the typical relative velocity between colliding grains as $\Delta V$, the impulse exerted at each collision scales as the grain mass multiplied by the velocity difference $\rho^{*} d^{3} \Delta V$. Collisions occur at a rate of $1 / t_{m f}$ and thus each grain exerts a force of $\rho^{*} d^{3} \Delta V / t_{m f}$. In dense flow, the number of grains per unit area scales as $1 / d^{2}$, so the pressure in the shear cell scales as

$$
p \sim \frac{\rho^{*} d \Delta V}{t_{m f}} .
$$

We suppose that the collision velocity $\Delta V$ is set by the fluctuation velocity of the grains $\delta v$ (that is, the square root of granular temperature $T^{1 / 2}$, as in Goldhirsch 2008), rather than the bulk shear rate. The fluctuation velocity is a further output of the system so, from dimensional analysis, it must take the form

$$
\delta v=d \dot{\gamma} \mathcal{H}\left(I, B, \phi_{r}, \mu, \varepsilon\right),
$$

for some function $\mathcal{H}$. As shown in figure $3 b$, the velocity fluctuations in our simulations collapse, following a power-law scaling with the inertial number, $\delta v /(d \dot{\gamma}) \sim I^{-1 / 4}$ for $I \lesssim$ 0.5 , but becoming dependent on grain contact properties at higher inertial numbers, which is in accordance with the modified kinetic theory predictions of Chialvo \& Sundaresan (2013). Substituting this scaling into (3.10), and rearranging for the mean free time then gives

$$
t_{m f} \sim \frac{d^{2} \dot{\gamma}}{p / \rho^{*}} I^{-1 / 4}=\frac{1}{\dot{\gamma}} I^{7 / 4} .
$$

Finally, under the applied acceleration $b_{r}$, a rising grain in free space for the time $t_{m f}$ between each collision gains an additional displacement $b_{r} t_{m f}{ }^{2} / 2$ in the direction of the applied acceleration, giving a percolation velocity

$$
w_{p r} \sim \frac{b_{r} t_{m f}^{2}}{t_{m f}} \sim \frac{b_{r}}{\dot{\gamma}} I^{7 / 4} .
$$

This scaling prediction for the percolation velocity has precisely the form (3.8) inferred from the numerical simulation results (figure $3 c$ ) in the dense granular flow regime $I \lesssim$ 0.5 .

\subsection{Effect of varying grain sizes}

Granular segregation is often driven by a size difference between grains. In order to test the validity of the drag law (3.13) for size-bidisperse grains, we perform the shear cell 


$\begin{array}{lccccccc}s & 1 & 1.05 & 1.11 & 1.25 & 1.43 & 1.67 & 2 \\ \Phi_{c} & 0.587 & 0.588 & 0.589 & 0.591 & 0.596 & 0.603 & 0.612\end{array}$

Table 2. Critical packing fraction as a function of grain size ratio $s$ for equal volume mixtures of bidisperse grains, with friction coefficient $\mu=0.5$.
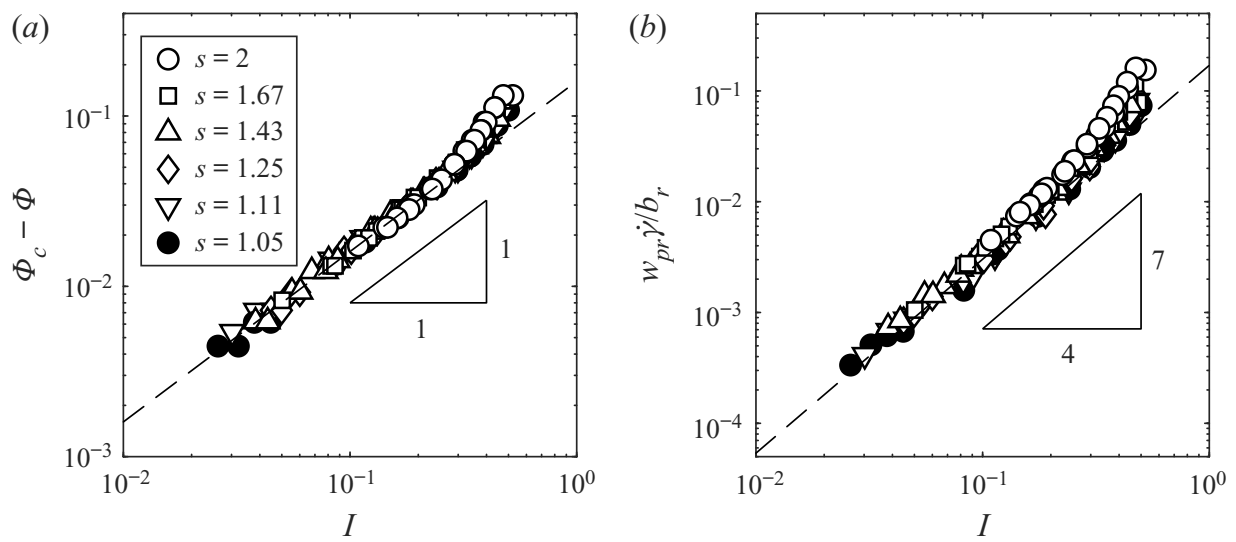

Figure 5. (a) Distance from critical packing fraction and $(b)$ non-dimensionalised percolation velocity against inertial number for size-bidisperse grains at a range of size ratios. Dashed lines are as in figures 3(a) and 3(c). Here, $\phi_{r}=0.5, \mu=0.5, \varepsilon=0.8$.

simulations using different grain sizes for the rising and falling grains. Due to the absence of any spatial gradients, the size difference between the two species does not result in segregating forces and, as before, the relative motion of the two species is driven entirely by the applied buoyancy force.

Bidispersity introduces a new dimensionless parameter, the grain size ratio, $s=$ $d_{r} / d_{f}$, where $d_{r, f}$ are the diameters of the rising and falling grains respectively. We examine mixtures where the difference between grain sizes is modest, between $s=1$ (monodisperse grains) and $s=2$.

To account for the different grain sizes, we modify the definition of the inertial number to be in terms of a volume-weighted mean grain diameter (Tripathi \& Khakhar 2011)

$$
\bar{d}=\phi_{r} d_{r}+\phi_{f} d_{f} .
$$

This definition of average diameter (3.14) is chosen because, out of the various definitions studied by $\mathrm{Gu}$, Ozel \& Sundaresan (2016), it gives the closest agreement between the rheology of the bidisperse mixture and the rheology of a monodisperse flow of grain size $\bar{d}$. The inertial number is then defined as

$$
I=\frac{\bar{d} \dot{\gamma}}{\sqrt{p / \rho^{*}}}
$$

which reduces to the monodisperse definition (3.6) when $s=1$.

Size-bidisperse grains are able to pack more densely than monodisperse grains, meaning that the critical packing fraction becomes dependent on the size ratio and mixture composition for the bidisperse grains, $\Phi_{c}=\Phi_{c}\left(\mu, s, \phi_{r}\right)$ (Desmond \& Weeks 2014). This function can be measured numerically as the solids volume fraction at which $I \rightarrow 0$; 


\section{Drag forces in granular segregation}

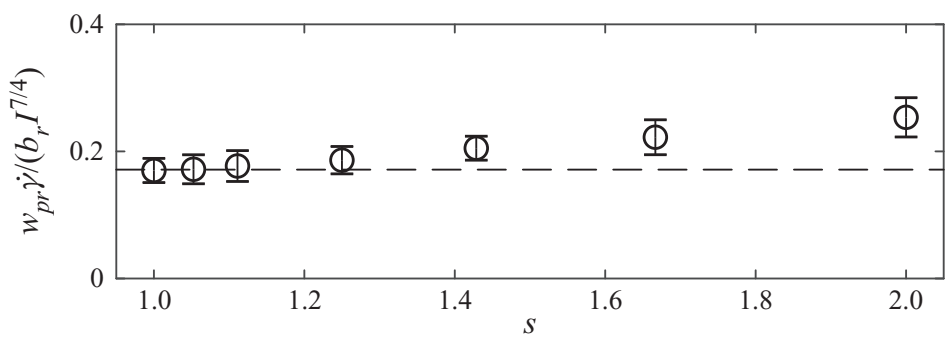

Figure 6. Dependence of percolation velocity on size ratio between the rising and falling species. Error bars represent the standard deviation of percolation velocity measurements presented in figures 4 and $5(b)$. Dashed line shows $\kappa=0.17$ as in figure 4 .

our measurements are given in table 2. Evaluating $\Phi_{c}$ in this way, the inertial number for bidisperse grains (3.15) then collapses onto exactly the same linear relationship with volume fraction (3.6) as was observed for monodisperse grains (figure $5 a$ ). This collapse, which is expected from the results of $\mathrm{Gu}$ et al. (2016), demonstrates that the modified inertial number (3.15) is dependent only on $\Phi_{c}-\Phi$, for the grain size ratios used here. Consequently, as with monodisperse grains, $I$ can substitute for $\Phi$ as a dimensionless parameter of the bidisperse system.

The percolation velocity of the grains is then found to observe the same $I^{7 / 4}$ scaling with inertial number (3.8) as monodisperse grains (figure $5 b$ ), when $I \lesssim 0.2$. For the larger particle size ratios studied, deviation of the measured percolation velocity from the $I^{7 / 4}$ scaling occurs when $I \gtrsim 0.2$, reflecting the nonlinearity in the relationship between $I$ and the underlying dimensionless parameter $\Phi$ that we observe for these relatively rapid bidisperse flows (figure $5 a$ ).

The drag coefficient $\kappa$ now varies slightly for different grain size ratios (figure 6 ), and may depend also on $\phi_{r}$ when $s \neq 1$. As expected, as $s \rightarrow 1$ we recover the same drag coefficient as for the monodisperse case, which is independent of $\phi_{r}$ (figure 4). When $\phi_{r}=1 / 2$, the system with grain size ratio $s$ is equivalent to that with size ratio $1 / s$, and consequently, the drag coefficient $\kappa$ must be an even function of $\log (s)$. For small grain size ratios $(|s-1| \ll 1)$ the change in $\kappa$ from its monodisperse value is therefore very small, of order $(s-1)^{2}$, which is consistent with the measurements in figure 6 . At larger size ratios the grains percolate more quickly under the same applied force, indicating a weakening of the drag as the grain size ratio increases.

\subsection{Diffusion}

The final contribution to the motion of a granular species in the force balance (1.2) is the diffusion. Although the uniform conditions of our shear cell mean that there is no net diffusive flux, diffusive processes are still active due to the fluctuating motion of grains. In the case of Fickian diffusion, the diffusivity $D_{j}$ in a coordinate direction $j$ is related to the motion of the grains through the relationship $\left\langle\left(\Delta x_{j}\right)^{2}\right\rangle=2 D_{j} t$, where $\left\langle\left(\Delta x_{j}\right)^{2}\right\rangle$ is the mean squared displacement in the $j$ th direction acquired by a grain in a time $t$. Dimensional analysis requires that each component of the diffusivity tensor takes the form

$$
D_{j}=d^{2} \dot{\gamma} \mathcal{D}_{j}\left(I, B, \phi_{r}, \mu, \varepsilon\right),
$$

for some dimensionless functions $\mathcal{D}_{j}$. Like the drag coefficient, the diffusivity is non-zero even in absence of a segregation force, and we do not observe any dependence of $D_{j}$ on the buoyancy strength $B$ or the fraction of rising grains $\phi_{r}$ for the values of $\tilde{B}$ used in this paper. The dependence of $D_{j}$ on shear rate and grain size has been observed 

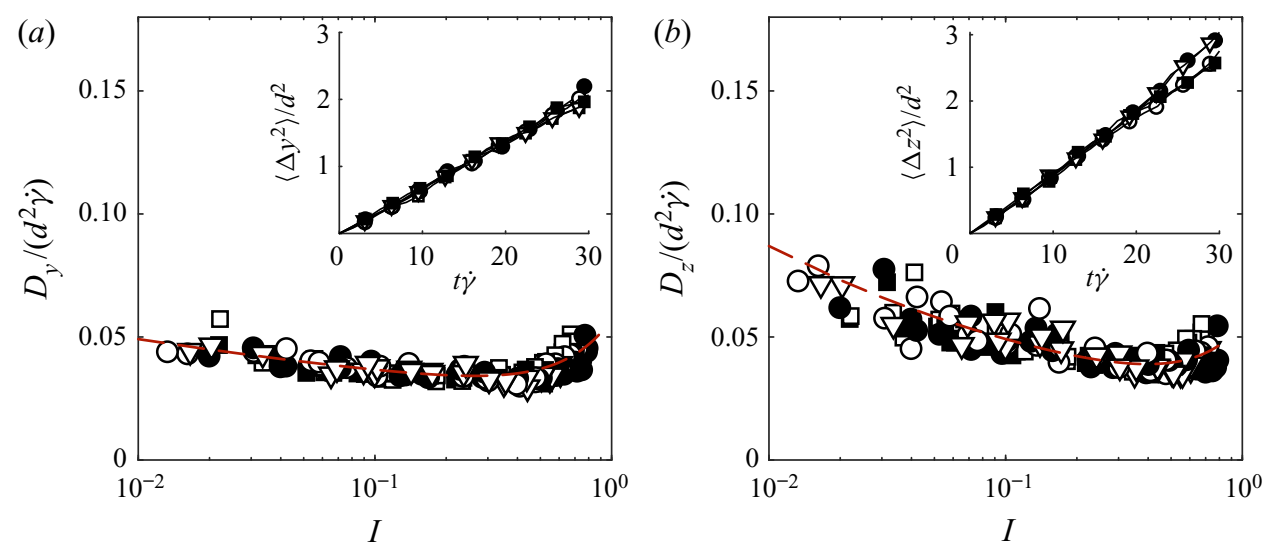

Figure 7. Measurements of the diffusivity in the $(a) y$-and $(b) z$-directions against inertial number. Symbol shapes and colours indicate contact-law parameters, as in figure 3, and red dashed lines show the empirical fitted curves (3.17) and (3.18). Insets: mean squared displacement against dimensionless time. $\phi_{r}=1 / 2$.

numerically (Campbell 1997) and experimentally (Utter \& Behringer 2004). However, the dependence of $D_{j}$ on inertial number has not yet been the subject of detailed study. We measure the mean squared displacement of the grains in our simulations in the $y$ direction, perpendicular to both the shearing and segregating flows (figure $7 a$ ), and in the $z$ direction, aligned with the segregation (figure $7 b$ ). When measuring the mean squared displacement in the $z$ direction, the mean segregating motion is first subtracted off of the motion of each grain.

We find that both $\left\langle\Delta y^{2}\right\rangle$ and $\left\langle\Delta z^{2}\right\rangle$ increase linearly with dimensionless time (figure 7 , insets), which validates the Fickian diffusion term in (1.2) and allows us to plot the diffusion coefficient as a function of inertial number (figure 7). We observe a small anisotropy in the diffusion with $D_{z}>D_{y}$. The measured diffusivities show only a weak dependence on inertial number, although, as observed by Campbell (1997), the diffusivity increases for $I \gtrsim 0.5$, outside the dense inertial flow regime. Over the range $0.01 \lesssim I \lesssim$ 0.8 , the measured diffusion coefficients are well approximated by the empirical functions

$$
\begin{gathered}
D_{y} /\left(d^{2} \dot{\gamma}\right)=\mathcal{D}_{y}=0.027 I^{-0.13}+0.03 I^{2} \approx 0.04, \\
D_{z} /\left(d^{2} \dot{\gamma}\right)=\mathcal{D}_{z}=0.0275 I^{-0.25}+0.0275 I^{2} \approx 0.05,
\end{gathered}
$$

as plotted on figure 7 . Over the range of $I$ considered, $\mathcal{D}_{z} \approx 0.05$ is almost constant, consistent with values found by Bridgwater (1980) and Savage \& Dai (1993) using experiments and simulations, respectively. The diffusion coefficient $\mathcal{D}_{z} \approx 0.05$ is also consistent with the values found by Cai et al. (2019) for size-bidisperse grains. Using the volume-weighted mean grain diameter $\bar{d}$ (3.14) in place of $d$, they find that the average diffusion coefficient is constant up to grain size ratios of $s=3$. Tripathi \& Khakhar (2013) demonstrate similarly that this coefficient maintains the same value in density-bidisperse mixtures, up to a density ratio of 3 .

\section{Implications for segregation in free-surface flows}

We now return to models for granular segregation, and examine the implications of our drag and diffusion scalings (3.9), (3.17), (3.18) for segregation of bidisperse grains in a free-surface flow. We consider the configuration of a shear flow $\boldsymbol{u}=u(z) \hat{\boldsymbol{x}}$, uniform in $x$, 


\section{Drag forces in granular segregation}

with segregation acting in the $z$ direction perpendicular to the flow, such that $S_{i}=S_{i} \hat{z}$, and $\boldsymbol{u}_{s i}=w_{i} \hat{z}$. This configuration is widely seen in segregation experiments, both in shear cells and free-surface flows. The uniaxial shear flow locally resembles the uniform simple shear used in the previous section, but allows pressure and shear rate to vary in $z$, providing the spatial gradients needed to drive segregation. For simplicity, we here consider that all grains are of the same intrinsic density $\rho^{*}$ but of varying sizes.

Under these conditions, the force balance (1.2) can be solved for the flux of the $i$ th species

$$
\phi_{i} w_{i}=\frac{S_{i}}{\rho^{*} c}-D_{z} \frac{\partial \phi_{i}}{\partial z} .
$$

Since the local dependence of $c$ and $D_{z}$ have been determined using the shear cell simulations, the segregating force $S_{i}$ is the only unmeasured quantity remaining in this equation. Substituting the flux (4.1) into the $z$ component of the mass balance (1.1), expanding the coefficients $c$ and $D_{z}$ using (3.9) and (3.18), and writing the inertial number explicitly gives the equation describing the segregation of the $i$ th species,

$$
\frac{\partial \phi_{i}}{\partial t}+\frac{\partial}{\partial z}\left[\kappa \frac{\dot{\gamma}^{3 / 4} \bar{d}^{7 / 4}}{\left(p^{7 / 8} / \rho^{* 7 / 8}\right)} \frac{S_{i}}{\rho^{*}}-\mathcal{D}_{z} \bar{d}^{2} \dot{\gamma} \frac{\partial \phi_{i}}{\partial z}\right]=0 .
$$

The first term within the square brackets is the segregation flux term $S_{i} / \rho^{*} c$. This flux remains completely general, as we have not specified the driving segregation force $S_{i}$ or its dependence on any of the flow variables. However, by comparing our expression for segregation flux to measurements of this flux and its scaling with $\dot{\gamma}$ and $p$, previously obtained through experiments or DEM simulations, we are able to suggest the dependence of the driving segregation force $S_{i}$ on these parameters. We focus on a single flow configuration, namely dense free-surface granular flows, for which there have been a number of studies measuring the segregation rate.

Several authors (e.g. May et al. 2010; Marks et al. 2012; Fan et al. 2014) propose that in free-surface flows the segregation flux is linear in shear rate $\dot{\gamma}$, based on measurements from experiments or discrete element simulations. Marks et al. (2012) attribute this linear dependence on $\dot{\gamma}$ to the segregation force $S_{i}$ alone, and suppose the drag coefficient $c$ is constant. From (4.2), our measurements of $c$ indicate that the dependence of drag on shear rate accounts for a scaling of the segregation flux as $\dot{\gamma}^{3 / 4}$, suggesting that the remaining dependence may be accounted for by relatively weak variation of the segregation force as $S_{i} \sim \dot{\gamma}^{1 / 4}$.

The segregation flux is observed to decrease with increasing pressure (e.g. Golick \& Daniels 2009; Fry et al. 2018), with recent measurements suggesting that the segregation flux that scales approximately as $p^{-1}$ (Duan et al. 2020; Barker et al. 2021). This is very close to the scaling $p^{-7 / 8}$ that we predict due to the dependence of just the drag on pressure, suggesting again that the driving segregation force $S_{i}$ depends only very weakly on pressure $\left(S_{i} \sim p^{-1 / 8}\right)$ in these flows.

This inference that the driving segregation force $S_{i}$ is nearly independent of $\dot{\gamma}$ and $p$ in free-surface flows is consistent with previous research. In steady free-surface flows, the (hydrostatic) pressure gradient is approximately constant due to the constant gravitational acceleration and near-constant grain volume fraction, resulting in a driving segregation force that is nearly independent of shear rate and pressure (Gray \& Thornton 2005). Guillard et al. (2016) report that this constant gravitational force is the largest driver of segregation in steady dense free-surface flows on an incline. The segregation force $S_{i}$ also depends on the gradient of granular temperature, which might be expected to depend on $\dot{\gamma}$ and $p$. However, Larcher \& Jenkins (2015) show that the specific granular temperature 


\section{R.S.J. Bancroft and C.G. Johnson}

distribution established in an equilibrium dense free-surface granular flow results in the driving force for kinetic-stress segregation scaling the same way as the gravitational segregation force. Thus, the near-constant driving segregation force that we deduce for free-surface flows is consistent with either segregation mechanism.

Typically, granular segregation rates have been inferred from experiments and discrete element simulations by measuring the changing packing fractions $\phi_{i}$ either in a simple uniaxial flow that is time dependent (e.g. Tunuguntla et al. 2014) or in a heap flow in which the flow field is steady, but non-uniaxial and varying in two spatial dimensions (e.g. Fan et al. 2014). It can be difficult or computationally expensive to relate the segregation rates measured to the flow conditions ( $\dot{\gamma}, p, \nabla T$ etc.), either because feedback of the changing flow composition on the rheology means that underlying flow evolves in time with the segregation (Kowalski \& McElwaine 2013), or because a large simulation domain is required to resolve a flow field that varies in two dimensions (Schlick et al. 2015). The characterisation of diffusion in (3.18) provides an alternative means of determining the driving segregation force $S_{i}$ from uniaxial shear flows that are steady and vary in only one spatial dimension. In a shear flow, the no-flux boundary conditions applied to the surface and base of a flow imply that the segregation flux (4.1) is zero throughout the flow (Gray \& Chugunov 2006). Consequently, (4.2) reduces to a one-dimensional balance between segregation and diffusion,

$$
S_{i}=\frac{\mathcal{D}_{z}}{\kappa}\left(\rho^{*} \bar{d}^{2} \dot{\gamma}^{2} p^{7}\right)^{1 / 8} \frac{\partial \phi_{i}}{\partial z} .
$$

In discrete element simulations, such a statistically steady state, that varies in only one spatial dimension, allows the quantities $p, \dot{\gamma}$ and $\partial \phi_{i} / \partial z$ to be measured to high precision, given sufficient simulation time, providing an accurate way to measure the driving segregation force.

\section{Conclusions}

We have made use of a steady uniform shear flow to study the drag force and diffusivity, which form a central part of mixture theory segregation models. Using discrete element simulations and dimensional analysis, the dependence of each of these on local flow properties has been found. In particular, for dense flows in the inertial regime, the coefficient of drag follows a power law in the inertial number with $c \sim \dot{\gamma} I^{-7 / 4}$, and we offer a simple model which provides an explanation for this scaling.

Our model highlights the role that velocity fluctuations have in setting the inter-species drag. Gradients in the grain fluctuation velocity or granular temperature are known to act as a driving force for segregation (Hill \& Tan 2014; Staron \& Phillips 2015); we show here that fluctuations also play a role in setting the speed of segregation through the drag force. Such fluctuations act to fluidise the flow, and consequently play an important role in the rheology (Kim \& Kamrin 2020), as well as the segregation of granular materials.

The approach used in this paper, namely studying the inter-species drag separately from the driving segregation forces, has several advantages over the approach of measuring the overall segregation flux in a non-uniform segregating flow. Since drag can be studied in a uniform environment, dimensional analysis can be used to constrain its parametric dependence, which in dense inertial flows reduces to a power-law scaling of a single dimensionless parameter $I$. Uniform environments also allow parameters to be measured in discrete element simulations with modest computational cost, resulting in very little scatter in the measured segregation velocity (figure $3 c$ ) and the consequent ability to constrain precise scaling relationships such as (3.8). 


\section{Drag forces in granular segregation}

Such improvements in the characterisation of segregation are much needed, since while segregation of polydisperse spheres of a modest range of sizes has been described (e.g. Marks et al. 2012; Schlick et al. 2016), modelling the segregation of highly polydisperse flows (with grain diameters ranging over several orders of magnitude) that occur in nature and industry remains a significant challenge. A wide range of grain sizes may result in complex demixing instabilities (Gray \& Ancey 2011), and permits additional segregation mechanisms such as spontaneous percolation (Wilkinson \& Edwards 1982), in addition to the shear-rate-dependent segregation discussed in this paper. Description of the grain size and density distributions introduces several additional non-dimensional parameters into the problem, but the methods proposed here extend naturally to allow characterisation of the drag in these complex polydisperse mixtures.

Declaration of interest. The authors report no conflict of interest.

\section{Author ORCIDs.}

(1) Robbie S.J. Bancroft https://orcid.org/0000-0002-2967-7731;

(1) Chris G. Johnson https://orcid.org/0000-0003-2192-3616.

\section{REFERENCES}

Ahmad, K. \& SMALley, I.J. 1973 Observation of particle segregation in vibrated granular systems. Powder Technol. 8 (1), 69-75.

BAGNOLD, R.A. 1954 Experiments on a gravity-free dispersion of large solid spheres in a Newtonian fluid under shear. Proc. R. Soc. Lond. A 225 (1160), 49-63.

Barker, T., Rauter, M., Maguire, E.S.F., Johnson, C.G. \& Gray, J.M.N.T. 2021 Coupling rheology and segregation in granular flows. J. Fluid Mech. 909, A22.

Baxter, J., TÜzÜn, U., Heyes, D., Hayati, I. \& Fredlund, P. 1998 Stratification in poured granular heaps. Nature 391 (6663), 136-136.

BRIDGWATER, J. 1980 Self-diffusion coefficients in deforming powders. Powder Technol. 25 (1), 129-131.

CAI, R., XiAO, H., ZHENG, J. \& ZHAO, Y. 2019 Diffusion of size bidisperse spheres in dense granular shear flow. Phys. Rev. E 99, 032902.

CAMPBell, C. 1997 Self-diffusion in granular shear flows. J. Fluid Mech. 348, 85-101.

Chassagne, R., Maurin, R., Chauchat, J., Gray, J.M.N.T. \& Frey, P. 2020 Discrete and continuum modelling of grain size segregation during bedload transport. J. Fluid Mech. 895, A30.

Chialvo, S., Sun, J. \& Sundaresan, S. 2012 Bridging the rheology of granular flows in three regimes. Phys. Rev. E 85, 021305.

Chialvo, S. \& Sundaresan, S. 2013 A modified kinetic theory for frictional granular flows in dense and dilute regimes. Phys. Fluids 25 (7), 070603.

da Cruz, F., Emam, S., Prochnow, M., Roux, J. \& Chevoir, F. 2005 Rheophysics of dense granular materials: discrete simulation of plane shear flows. Phys. Rev. E 72, 021309.

CUNDALL, P.A. \& STRACK, O.D.L. 1979 A discrete numerical model for granular assemblies. Géotechnique 29 (1), 47-65.

Desmond, K.W. \& WeEKS, E.R. 2014 Influence of particle size distribution on random close packing of spheres. Phys. Rev. E 90, 022204.

Duan, Y., Umbanhowar, P.B., Ottino, J.M. \& Lueptow, R.M. 2020 Segregation models for density-bidisperse granular flows. Phys. Rev. Fluids 5 (4), 044301.

Fan, Y., Schlick, C.P., Umbanhowar, P.B., Ottino, J.M. \& Lueptow, R.M. 2014 Modelling size segregation of granular materials: the roles of segregation, advection and diffusion. J. Fluid Mech. 741, 252-279.

Forterre, Y. \& Pouliquen, O. 2008 Flows of dense granular media. Annu. Rev. Fluid Mech. 40, 1-24.

Fry, A.M., Umbanhowar, P.B., Ottino, J.M. \& LuEPtow, R.M. 2018 Effect of pressure on segregation in granular shear flows. Phys. Rev. E 97, 062906.

GDR-MIDi 2004 On dense granular flows. Eur. Phys. J. E 14 (4), 341-365.

GoldhiRsCH, I. 2008 Introduction to granular temperature. Powder Technol. 182 (2), 130-136.

GoliCK, L.A. \& DANIELS, K.E. 2009 Mixing and segregation rates in sheared granular materials. Phys. Rev. E 80 (4), 042301.

GRAY, J.M.N.T. \& ANCEY, C. 2011 Multi-component particle-size segregation in shallow granular avalanches. J. Fluid Mech. 678, 535-588. 
Gray, J.M.N.T. \& Chugunov, V.A. 2006 Particle-size segregation and diffusive remixing in shallow granular avalanches. J. Fluid Mech. 569, 365-398.

Gray, J.M.N.T. \& HUtTER, K. 1997 Pattern formation in granular avalanches. Contin. Mech. Thermodyn. 9 (6), 341-345.

Gray, J.M.N.T \& THORnTON, A.R. 2005 A theory for particle size segregation in shallow granular free-surface flows. Proc. R. Soc. Lond. A 461 (2057), 1447-1473.

Gu, Y., Ozel, A. \& Sundaresan, S. 2016 Rheology of granular materials with size distributions across dense-flow regimes. Powder Technol. 295, 322-329.

Guillard, F., Forterre, Y. \& Pouliquen, O. 2016 Scaling laws for segregation forces in dense sheared granular flows. J. Fluid Mech. 807, R1.

HILL, K.M. \& TAN, D.S. 2014 Segregation in dense sheared flows: gravity, temperature gradients, and stress partitioning. J. Fluid Mech. 756, 54-88.

Jing, L., OtTino, J.M., LuePtow, R.M. \& Umbanhowar, P.B. 2020 Rising and sinking intruders in dense granular flows. Phys. Rev. Res. 2, 022069.

Johnson, C.G., Kokelaar, B.P., Iverson, R.M., Logan, M., LaHusen, R.G. \& Gray, J.M.N.T. 2012 Grain-size segregation and levee formation in geophysical mass flows. J. Geophys. Res.: Earth 117, F01032.

Kim, S. \& KAMRin, K. 2020 Power-law scaling in granular rheology across flow geometries. Phys. Rev. Lett. 125, 088002.

KowALSKI, J. \& MCElwaine, J.N. 2013 Shallow two-component gravity-driven flows with vertical variation. J. Fluid Mech. 714, 434-462.

LARCHER, M. \& JENKINS, J. 2015 The evolution of segregation in dense inclined flows of binary mixtures of spheres. J. Fluid Mech. 782, 405-429.

LEES, A.W. \& EDWARDS, S.F. 1972 The computer study of transport processes under extreme conditions. J. Phys. C 5 (15), 1921-1928.

MARKS, B., RognON, P. \& EinAV, I. 2012 Grainsize dynamics of polydisperse granular segregation down inclined planes. J. Fluid Mech. 690, 499-511.

May, L.B.H., Golick, L.A., Phillips, K.C., Shearer, M. \& Daniels, K.E. 2010 Shear-driven size segregation of granular materials: modeling and experiment. Phys. Rev. E 81 (5), 051301.

SAVAGE, S.B. \& DAI, R. 1993 Studies of granular shear flows wall slip velocities, 'layering' and self-diffusion. Mech. Mater. 16 (1), 225-238, special Issue on Mechanics of Granular Materials.

Savage, S.B. \& Lun, C.K.K. 1988 Particle size segregation in inclined chute flow of dry cohesionless granular solids. J. Fluid Mech. 189, 311-335.

SChlick, C.P., Fan, Y., IsNer, A.B., Umbanhowar, P.B., Ottino, J.M. \& Lueptow, R.M. 2015 Modeling segregation of bidisperse granular materials using physical control parameters in the quasi-2D bounded heap. AIChE J. 61 (5), 1524-1534.

Schlick, C.P., Isner, A.B., Freireich, B.J., Fan, Y., Umbanhowar, P.B., Ottino, J.M. \& LUEPTOW, R.M. 2016 A continuum approach for predicting segregation in flowing polydisperse granular materials. J. Fluid Mech. 797, 95-109.

Silbert, L.E., Ertaş, D., Grest, G.S., Halsey, T.C., Levine, D. \& Plimpton, S.J. 2001 Granular flow down an inclined plane: Bagnold scaling and rheology. Phys. Rev. E 64, 051302.

STARON, L. \& PhILlipS, J.C. 2015 Stress partition and microstructure in size-segregating granular flows. Phys. Rev. E 92, 022210.

TRIPATHI, A. \& KHAKHAR, D.V. 2011 Rheology of binary granular mixtures in the dense flow regime. Phys. Fluids 23 (11), 113302.

Tripathi, A. \& KhaKhar, D.V. 2013 Density difference-driven segregation in a dense granular flow. J. Fluid Mech. 717, 643-669.

Tunuguntla, D.R., Bokhove, O. \& Thornton, A.R. 2014 A mixture theory for size and density segregation in shallow granular free-surface flows. J. Fluid Mech. 749, 99-112.

UtTER, B. \& BeHRinger, R.P. 2004 Self-diffusion in dense granular shear flows. Phys. Rev. E 69, 031308.

VAN DeR VAart, K., VAn Schrojenstein Lantman, M.P., Weinhart, T., Luding, S., AnCey, C. \& THORNTON, A.R. 2018 Segregation of large particles in dense granular flows suggests a granular Saffman effect. Phys. Rev. Fluids 3, 074303.

Weinhart, T., et al., 2020 Fast, flexible particle simulations - an introduction to MercuryDPM. Comput. Phys. Commun. 249, 107129.

Weinhart, T., Luding, S. \& Thornton, A. 2013 From discrete particles to continuum fields in mixtures. AIP Conf. Proc. 1542, 1202-1205.

WiLKINSON, D.R. \& EDWARDS, S.F. 1982 Spontaneous interparticle percolation. Proc. R. Soc. Lond. A 381 (1780), 33-51. 